\title{
Approach of Mental State Analysis using EEG
}

\author{
Chaitali Bhattacharyya*, Trisha Paul*, Susmita Das"*, Soutrik Karmakar", Shyam Sundar Banerjee* \\ *Electronics and Instrumentation Engg., Narula Institute of Technology \\ ${ }^{* *}$ Electronics and Instrumentation Engg., Narula Institute of Technology \\ DOI: 10.29322/IJSRP.10.02.2020.p9805 \\ http://dx.doi.org/10.29322/IJSRP.10.02.2020.p9805
}

\begin{abstract}
Electroencephalogram (EEG) signals from human brain have ground-breaking impact in the field of health care industry. EEG is one of the most actively used bio signal as measurement tool in Brain-Computer Interface (BCI) to implement "thoughts or intention" based human and machine interaction. In the medical field, EEG based interaction is also used for computer aided diagnosis for the patients and also to help in detecting emotions and body movements. In this paper, the beneficial aspect of EEG is demonstrated which can be the great help for paralysed patients. The study of characteristics of the signals while the person is moving and when the person is intending to move to be diagnosed through BCI can be controlled for humanoid movement through EEG.
\end{abstract}

Index Terms- EEG, BCI, HMI, Brain waves.

\section{INTRODUCTION}

$\mathrm{T}$ he Electroencephalogram (EEG) is a method known as an electrophysiology for monitoring and recording the electrical signal activity of the brain. This EEG read the electrical signal acquired from the scalp of the brain. EEG can give several types of clinical indications such as for the patients applied with anesthesia and observing the output waveforms of EEG to detect the consciousness level of the person [1]. Brain Computer Interface (BCI) [2] is the main focusing area for the advanced implementation of mental state prediction through Human Computer Interaction (HMI). The EEG [3] obey and maintain the differential voltage amplification principle where two types of bio-electrodes are used. One electrode is main or active electrode which collects the output of EEG and another part is reference electrode. The measurement unit of EEG is in microvolt with respect to the brain frequencies i.e. delta, theta, alpha, beta frequencies.

\section{METHODOLOGY}

In this study, especially the consciousness of human beings through analysis of the values of EEG [6] to predict the physical condition of that particular human is observed. By analyzing the EEG values the prediction of the particular physical fitness of human being can be detected. The most important and functional [7] organ is brain. Brain works as the coordinator and controller of the muscles and nerves in the human being's body. Different parts of the brain control [8] different actions. Cerebellum is located behind the top part of the brain and it is responsible for the movements, balance and muscular activities. The specialized cells in Cerebellum transmits information via electrical signal [9]. As different nerves are responsible for different functions, it is important to know the functional behavior and the location of the coronial nerves to place the electrodes for EEG [10][11] recordings.

Table 1: Brain Wave Classification [5]

\begin{tabular}{|c|c|c|}
\hline SL. No. & Brain-Wave Type & Frequency $(\mathrm{Hz})$ \\
\hline 1 & Delta Wave & $0-4$ \\
\hline 2 & Alpha wave & $8-12$ \\
\hline 3 & Theta wave & $4-8$ \\
\hline 4 & Gamma Wave & $40-100$ \\
\hline 5 & Beta & $12-40$ \\
\hline
\end{tabular}

Brain waves are detected by the sensors placed on the scalp. The signal changes according to the level of concentration. If the brain wave has lower frequency means at drowsy state and higher frequency brain waves means in focused condition. Brain waves are usually sinusoidal and measured from peak to peak which normally range from 0.5 to $100 \mu \mathrm{V}$. 
III. Results and Discussions

The output measurements are taken from esense meter present within the brain signal collecting device used in this work.

Table 2: Concentration Level Measurement Data [4]

\begin{tabular}{|c|c|c|c|c|c|c|c|c|c|c|c|c|}
\hline $\begin{array}{c}\text { Time } \\
\text { (mse } \\
\text { c) }\end{array}$ & $\begin{array}{c}\text { Esense } \\
\text { output } \\
(\mathrm{mV})\end{array}$ & $\begin{array}{c}\text { Esense } \\
\text { output } \\
(\mathrm{mV})\end{array}$ & $\begin{array}{c}\text { Esense } \\
\text { output } \\
(\mathrm{mV})\end{array}$ & $\begin{array}{c}\text { Esense } \\
\text { output } \\
(\mathrm{mV})\end{array}$ & $\begin{array}{c}\text { low } \\
\text { gamma } \\
(\mathrm{Hz})\end{array}$ & $\begin{array}{c}\text { high } \\
\text { gamma } \\
(\mathrm{Hz})\end{array}$ & $\begin{array}{c}\text { high } \\
\text { alpha } \\
(\mathrm{Hz})\end{array}$ & $\begin{array}{c}\text { low } \\
\text { alpha } \\
(\mathrm{Hz})\end{array}$ & $\begin{array}{l}\text { high } \\
\text { beta } \\
(\mathrm{Hz})\end{array}$ & $\begin{array}{l}\text { low } \\
\text { beta } \\
(\mathrm{Hz})\end{array}$ & $\begin{array}{c}\text { Delta } \\
(\mathrm{Hz})\end{array}$ & $\begin{array}{c}\text { Theta } \\
(\mathrm{Hz})\end{array}$ \\
\hline $\begin{array}{l}0.16 \\
7812\end{array}$ & 0 & 0 & 90 & 84 & 1508 & 949 & 3203 & $\begin{array}{c}1551 \\
4\end{array}$ & 8016 & $\begin{array}{c}1014 \\
6\end{array}$ & $\begin{array}{c}1337 \\
36\end{array}$ & $\begin{array}{c}3869 \\
1\end{array}$ \\
\hline $\begin{array}{c}0.18 \\
0342\end{array}$ & 0 & 0 & 84 & 70 & 3783 & 2777 & 3485 & $\begin{array}{c}1076 \\
8 \\
\end{array}$ & 5870 & $\begin{array}{c}1814 \\
8 \\
\end{array}$ & $\begin{array}{c}5734 \\
95 \\
\end{array}$ & $\begin{array}{c}1838 \\
8 \\
\end{array}$ \\
\hline $\begin{array}{c}0.19 \\
3154\end{array}$ & 0 & 0 & 96 & 56 & 9032 & 7513 & $\begin{array}{c}1527 \\
9\end{array}$ & $\begin{array}{c}1407 \\
7\end{array}$ & $\begin{array}{c}1149 \\
6\end{array}$ & $\begin{array}{c}1312 \\
9\end{array}$ & $\begin{array}{c}1179 \\
111\end{array}$ & $\begin{array}{c}1720 \\
0\end{array}$ \\
\hline $\begin{array}{c}0.20 \\
5996\end{array}$ & 0 & 0 & 100 & 64 & 29317 & 7823 & $\begin{array}{c}2535 \\
1 \\
\end{array}$ & $\begin{array}{c}6155 \\
9 \\
\end{array}$ & $\begin{array}{c}2578 \\
0 \\
\end{array}$ & $\begin{array}{c}9200 \\
4 \\
\end{array}$ & $\begin{array}{c}4361 \\
23\end{array}$ & $\begin{array}{c}3775 \\
86 \\
\end{array}$ \\
\hline $\begin{array}{c}0.21 \\
689 \\
\end{array}$ & 0 & 0 & 100 & 75 & 4104 & 3243 & 3700 & 9609 & $\begin{array}{c}1069 \\
4\end{array}$ & $\begin{array}{c}1015 \\
2\end{array}$ & $\begin{array}{c}1084 \\
586\end{array}$ & $\begin{array}{c}3672 \\
6\end{array}$ \\
\hline $\begin{array}{c}0.22 \\
9007\end{array}$ & 0 & 0 & 100 & 66 & 10283 & 3179 & 8039 & 5281 & $\begin{array}{c}1285 \\
5 \\
\end{array}$ & $\begin{array}{c}1204 \\
5 \\
\end{array}$ & $\begin{array}{c}7044 \\
7 \\
\end{array}$ & $\begin{array}{c}3223 \\
9 \\
\end{array}$ \\
\hline $\begin{array}{c}0.24 \\
0852\end{array}$ & 0 & 0 & 100 & 61 & 33718 & 3740 & $\begin{array}{c}1055 \\
5\end{array}$ & $\begin{array}{c}2498 \\
6 \\
\end{array}$ & $\begin{array}{c}2550 \\
8 \\
\end{array}$ & $\begin{array}{c}2373 \\
2\end{array}$ & $\begin{array}{c}1293 \\
020\end{array}$ & $\begin{array}{c}1662 \\
94\end{array}$ \\
\hline $\begin{array}{c}0.25 \\
2404\end{array}$ & 0 & 0 & 90 & 37 & 1238 & 1470 & 124 & 2192 & 3295 & 937 & $\begin{array}{c}2808 \\
3 \\
\end{array}$ & $\begin{array}{c}1410 \\
4\end{array}$ \\
\hline $\begin{array}{l}0.26 \\
5383\end{array}$ & 0 & 0 & 100 & 40 & 28517 & 88436 & $\begin{array}{c}8034 \\
4\end{array}$ & $\begin{array}{c}3003 \\
5 \\
\end{array}$ & $\begin{array}{c}1922 \\
5\end{array}$ & $\begin{array}{c}2330 \\
9\end{array}$ & $\begin{array}{c}1436 \\
290\end{array}$ & $\begin{array}{c}1771 \\
30\end{array}$ \\
\hline $\begin{array}{l}0.27 \\
6735\end{array}$ & 0 & 0 & 91 & 54 & 4307 & 29775 & 4808 & $\begin{array}{c}1061 \\
7\end{array}$ & 3281 & $\begin{array}{c}1180 \\
9\end{array}$ & $\begin{array}{c}1148 \\
25\end{array}$ & $\begin{array}{c}2986 \\
7\end{array}$ \\
\hline $\begin{array}{c}0.28 \\
8082\end{array}$ & 0 & 0 & 69 & 61 & 11526 & 90991 & $\begin{array}{c}2005 \\
0\end{array}$ & $\begin{array}{c}3075 \\
5 \\
\end{array}$ & $\begin{array}{c}1700 \\
0 \\
\end{array}$ & 2993 & $\begin{array}{c}7277 \\
6 \\
\end{array}$ & $\begin{array}{c}8438 \\
3\end{array}$ \\
\hline $\begin{array}{c}0.29 \\
801 \\
\end{array}$ & 0 & 0 & 69 & 75 & 16287 & 77329 & $\begin{array}{c}3204 \\
8 \\
\end{array}$ & 8935 & $\begin{array}{c}1873 \\
2 \\
\end{array}$ & $\begin{array}{c}2097 \\
5 \\
\end{array}$ & $\begin{array}{c}1042 \\
86\end{array}$ & $\begin{array}{c}9500 \\
3\end{array}$ \\
\hline $\begin{array}{c}0.31 \\
089\end{array}$ & 0 & 0 & 64 & 83 & 15751 & 71115 & $\begin{array}{c}6387 \\
9 \\
\end{array}$ & 1378 & $\begin{array}{c}1129 \\
3 \\
\end{array}$ & $\begin{array}{c}1238 \\
8\end{array}$ & $\begin{array}{c}1365 \\
915 \\
\end{array}$ & $\begin{array}{c}5995 \\
8\end{array}$ \\
\hline $\begin{array}{c}0.32 \\
0636\end{array}$ & 0 & 0 & 69 & 90 & 6730 & 4119 & $\begin{array}{c}2159 \\
0\end{array}$ & $\begin{array}{c}1478 \\
0\end{array}$ & $\begin{array}{c}2302 \\
1\end{array}$ & 9032 & $\begin{array}{c}8075 \\
42\end{array}$ & $\begin{array}{c}1796 \\
03\end{array}$ \\
\hline $\begin{array}{c}0.33 \\
0916\end{array}$ & 0 & 0 & 77 & 74 & 4956 & 4589 & $\begin{array}{c}2531 \\
8\end{array}$ & 7055 & $\begin{array}{c}2646 \\
4\end{array}$ & 8370 & $\begin{array}{c}6483 \\
0\end{array}$ & 4086 \\
\hline $\begin{array}{c}0.34 \\
2872\end{array}$ & 0 & 0 & 90 & 77 & 938 & 928 & $\begin{array}{c}1049 \\
7\end{array}$ & $\begin{array}{c}1831 \\
9\end{array}$ & 4187 & 2472 & $\begin{array}{c}1206 \\
15\end{array}$ & $\begin{array}{c}5752 \\
0\end{array}$ \\
\hline $\begin{array}{c}0.35 \\
5476\end{array}$ & 0 & 0 & 70 & 84 & 1192 & 781 & $\begin{array}{c}2197 \\
2\end{array}$ & $\begin{array}{c}1161 \\
5\end{array}$ & 4556 & 6700 & $\begin{array}{c}1864 \\
09\end{array}$ & $\begin{array}{c}3688 \\
1\end{array}$ \\
\hline $\begin{array}{c}0.36 \\
7898\end{array}$ & 0 & 0 & 63 & 84 & 7839 & 3518 & $\begin{array}{c}1218 \\
3\end{array}$ & 6902 & 6836 & $\begin{array}{c}3023 \\
3 \\
\end{array}$ & $\begin{array}{c}1025 \\
026 \\
\end{array}$ & $\begin{array}{c}5301 \\
5 \\
\end{array}$ \\
\hline $\begin{array}{c}0.38 \\
0892\end{array}$ & 0 & 0 & 57 & 91 & 6580 & 3994 & 438 & $\begin{array}{c}9709 \\
6\end{array}$ & $\begin{array}{c}1751 \\
2\end{array}$ & $\begin{array}{c}4573 \\
0\end{array}$ & $\begin{array}{c}1874 \\
658\end{array}$ & $\begin{array}{c}1721 \\
74\end{array}$ \\
\hline
\end{tabular}



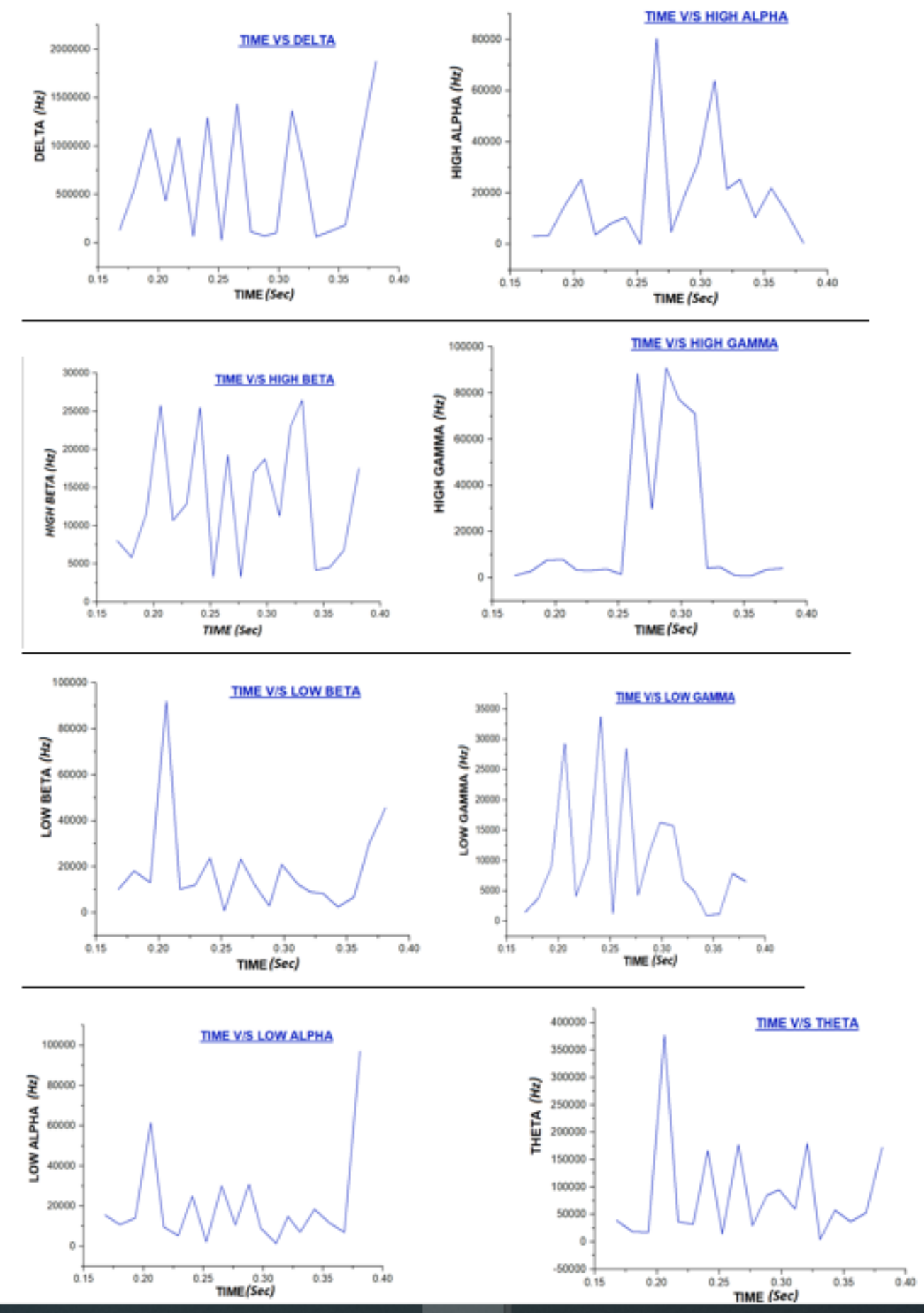

Fig. 1: Graphical output presentation of Concentration level data taken from human Brain

The mental state of the person has been observed from the values of low gamma, high gamma, high alpha, low alpha, high beta, low beta, delta and theta and the output graphs. The peak values of these graphs are greater than the standard values of each parameters. So, it can be predicted that the person's mental state is in concentrated mode. 
Table 3: Meditation Level Measurement Data [4]

\begin{tabular}{|c|c|c|c|c|c|c|c|c|c|c|c|c|}
\hline $\begin{array}{l}\text { Time } \\
\text { (mse } \\
\text { c) }\end{array}$ & $\begin{array}{c}\text { Esense } \\
\text { output } \\
(\mathrm{mV})\end{array}$ & $\begin{array}{c}\text { Esense } \\
\text { output } \\
(\mathrm{mV})\end{array}$ & $\begin{array}{c}\text { Esense } \\
\text { output } \\
(\mathrm{mV})\end{array}$ & $\begin{array}{c}\text { Esense } \\
\text { output } \\
(\mathrm{mV})\end{array}$ & $\begin{array}{c}\text { low } \\
\text { gamma } \\
(\mathrm{Hz})\end{array}$ & $\begin{array}{l}\text { high } \\
\text { gamma } \\
(\mathrm{Hz})\end{array}$ & $\begin{array}{l}\text { high } \\
\text { alpha } \\
(\mathrm{Hz})\end{array}$ & $\begin{array}{c}\text { low } \\
\text { alpha } \\
(\mathrm{Hz})\end{array}$ & $\begin{array}{l}\text { high } \\
\text { beta } \\
(\mathrm{Hz})\end{array}$ & $\begin{array}{l}\text { low } \\
\text { beta } \\
(\mathrm{Hz})\end{array}$ & $\begin{array}{c}\text { Delta } \\
(\mathrm{Hz})\end{array}$ & $\begin{array}{l}\text { Theta } \\
(\mathrm{Hz})\end{array}$ \\
\hline $\begin{array}{l}0.16 \\
7812\end{array}$ & 0 & 0 & 90 & 84 & 1508 & 949 & 3203 & $\begin{array}{c}1551 \\
4\end{array}$ & 8016 & $\begin{array}{c}1014 \\
6\end{array}$ & $\begin{array}{c}1337 \\
36\end{array}$ & $\begin{array}{c}3869 \\
1\end{array}$ \\
\hline $\begin{array}{c}0.18 \\
0342\end{array}$ & 0 & 0 & 84 & 70 & 3783 & 2777 & 3485 & $\begin{array}{c}1076 \\
8\end{array}$ & 5870 & $\begin{array}{c}1814 \\
8\end{array}$ & $\begin{array}{c}5734 \\
95\end{array}$ & $\begin{array}{c}1838 \\
8\end{array}$ \\
\hline $\begin{array}{l}0.19 \\
3154\end{array}$ & 0 & 0 & 96 & 56 & 9032 & 7513 & $\begin{array}{c}1527 \\
9\end{array}$ & $\begin{array}{c}1407 \\
7\end{array}$ & $\begin{array}{c}1149 \\
6\end{array}$ & $\begin{array}{c}1312 \\
9\end{array}$ & $\begin{array}{c}1179 \\
111\end{array}$ & $\begin{array}{c}1720 \\
0\end{array}$ \\
\hline $\begin{array}{c}0.20 \\
5996\end{array}$ & 0 & 0 & 100 & 64 & 29317 & 7823 & $\begin{array}{c}2535 \\
1\end{array}$ & $\begin{array}{c}6155 \\
9\end{array}$ & $\begin{array}{c}2578 \\
0\end{array}$ & $\begin{array}{c}9200 \\
4\end{array}$ & $\begin{array}{c}4361 \\
23\end{array}$ & $\begin{array}{c}3775 \\
86\end{array}$ \\
\hline $\begin{array}{l}0.21 \\
689\end{array}$ & 0 & 0 & 100 & 75 & 4104 & 3243 & 3700 & 9609 & $\begin{array}{c}1069 \\
4\end{array}$ & $\begin{array}{c}1015 \\
2\end{array}$ & $\begin{array}{c}1084 \\
586\end{array}$ & $\begin{array}{c}3672 \\
6\end{array}$ \\
\hline $\begin{array}{c}0.22 \\
9007\end{array}$ & 0 & 0 & 100 & 66 & 10283 & 3179 & 8039 & 5281 & $\begin{array}{c}1285 \\
5\end{array}$ & $\begin{array}{c}1204 \\
5\end{array}$ & $\begin{array}{c}7044 \\
7\end{array}$ & $\begin{array}{c}3223 \\
9\end{array}$ \\
\hline $\begin{array}{c}0.24 \\
0852\end{array}$ & 0 & 0 & 100 & 61 & 33718 & 3740 & $\begin{array}{c}1055 \\
5\end{array}$ & $\begin{array}{c}2498 \\
6\end{array}$ & $\begin{array}{c}2550 \\
8\end{array}$ & $\begin{array}{c}2373 \\
2\end{array}$ & $\begin{array}{c}1293 \\
020\end{array}$ & $\begin{array}{c}1662 \\
94\end{array}$ \\
\hline $\begin{array}{c}0.25 \\
2404\end{array}$ & 0 & 0 & 90 & 37 & 1238 & 1470 & 124 & 2192 & 3295 & 937 & $\begin{array}{c}2808 \\
3\end{array}$ & $\begin{array}{c}1410 \\
4\end{array}$ \\
\hline $\begin{array}{l}0.26 \\
5383\end{array}$ & 0 & 0 & 100 & 40 & 28517 & 88436 & $\begin{array}{c}8034 \\
4\end{array}$ & $\begin{array}{c}3003 \\
5\end{array}$ & $\begin{array}{c}1922 \\
5\end{array}$ & $\begin{array}{c}2330 \\
9\end{array}$ & $\begin{array}{l}1436 \\
290\end{array}$ & $\begin{array}{c}1771 \\
30\end{array}$ \\
\hline $\begin{array}{l}0.27 \\
6735\end{array}$ & 0 & 0 & 91 & 54 & 4307 & 29775 & 4808 & $\begin{array}{c}1061 \\
7\end{array}$ & 3281 & $\begin{array}{c}1180 \\
9\end{array}$ & $\begin{array}{c}1148 \\
25\end{array}$ & $\begin{array}{c}2986 \\
7\end{array}$ \\
\hline $\begin{array}{l}0.28 \\
8082\end{array}$ & 0 & 0 & 69 & 61 & 11526 & 90991 & $\begin{array}{c}2005 \\
0\end{array}$ & $\begin{array}{c}3075 \\
5\end{array}$ & $\begin{array}{c}1700 \\
0\end{array}$ & 2993 & $\begin{array}{c}7277 \\
6\end{array}$ & $\begin{array}{c}8438 \\
3\end{array}$ \\
\hline $\begin{array}{c}0.29 \\
801\end{array}$ & 0 & 0 & 69 & 75 & 16287 & 77329 & $\begin{array}{c}3204 \\
8\end{array}$ & 8935 & $\begin{array}{c}1873 \\
2\end{array}$ & $\begin{array}{c}2097 \\
5\end{array}$ & $\begin{array}{c}1042 \\
86\end{array}$ & $\begin{array}{c}9500 \\
3\end{array}$ \\
\hline $\begin{array}{c}0.31 \\
089\end{array}$ & 0 & 0 & 64 & 83 & 15751 & 71115 & $\begin{array}{c}6387 \\
9\end{array}$ & 1378 & $\begin{array}{c}1129 \\
3\end{array}$ & $\begin{array}{c}1238 \\
8\end{array}$ & $\begin{array}{c}1365 \\
915\end{array}$ & $\begin{array}{c}5995 \\
8\end{array}$ \\
\hline $\begin{array}{c}0.32 \\
0636\end{array}$ & 0 & 0 & 69 & 90 & 6730 & 4119 & $\begin{array}{c}2159 \\
0\end{array}$ & $\begin{array}{c}1478 \\
0\end{array}$ & $\begin{array}{c}2302 \\
1\end{array}$ & 9032 & $\begin{array}{c}8075 \\
42\end{array}$ & $\begin{array}{c}1796 \\
03\end{array}$ \\
\hline $\begin{array}{c}0.33 \\
0916\end{array}$ & 0 & 0 & 77 & 74 & 4956 & 4589 & $\begin{array}{c}2531 \\
8\end{array}$ & 7055 & $\begin{array}{c}2646 \\
4\end{array}$ & 8370 & $\begin{array}{c}6483 \\
0\end{array}$ & 4086 \\
\hline $\begin{array}{c}0.34 \\
2872\end{array}$ & 0 & 0 & 90 & 77 & 938 & 928 & $\begin{array}{c}1049 \\
7\end{array}$ & $\begin{array}{c}1831 \\
9\end{array}$ & 4187 & 2472 & $\begin{array}{c}1206 \\
15\end{array}$ & $\begin{array}{c}5752 \\
0\end{array}$ \\
\hline $\begin{array}{c}0.35 \\
5476\end{array}$ & 0 & 0 & 70 & 84 & 1192 & 781 & $\begin{array}{c}2197 \\
2\end{array}$ & $\begin{array}{c}1161 \\
5\end{array}$ & 4556 & 6700 & $\begin{array}{c}1864 \\
09\end{array}$ & $\begin{array}{c}3688 \\
1\end{array}$ \\
\hline $\begin{array}{l}0.36 \\
7898\end{array}$ & 0 & 0 & 63 & 84 & 7839 & 3518 & $\begin{array}{c}1218 \\
3\end{array}$ & 6902 & 6836 & $\begin{array}{c}3023 \\
3\end{array}$ & $\begin{array}{c}1025 \\
026\end{array}$ & $\begin{array}{c}5301 \\
5\end{array}$ \\
\hline $\begin{array}{c}0.38 \\
0892\end{array}$ & 0 & 0 & 57 & 91 & 6580 & 3994 & 438 & $\begin{array}{c}9709 \\
6\end{array}$ & $\begin{array}{c}1751 \\
2\end{array}$ & $\begin{array}{c}4573 \\
0\end{array}$ & $\begin{array}{c}1874 \\
658\end{array}$ & $\begin{array}{c}1721 \\
74\end{array}$ \\
\hline
\end{tabular}



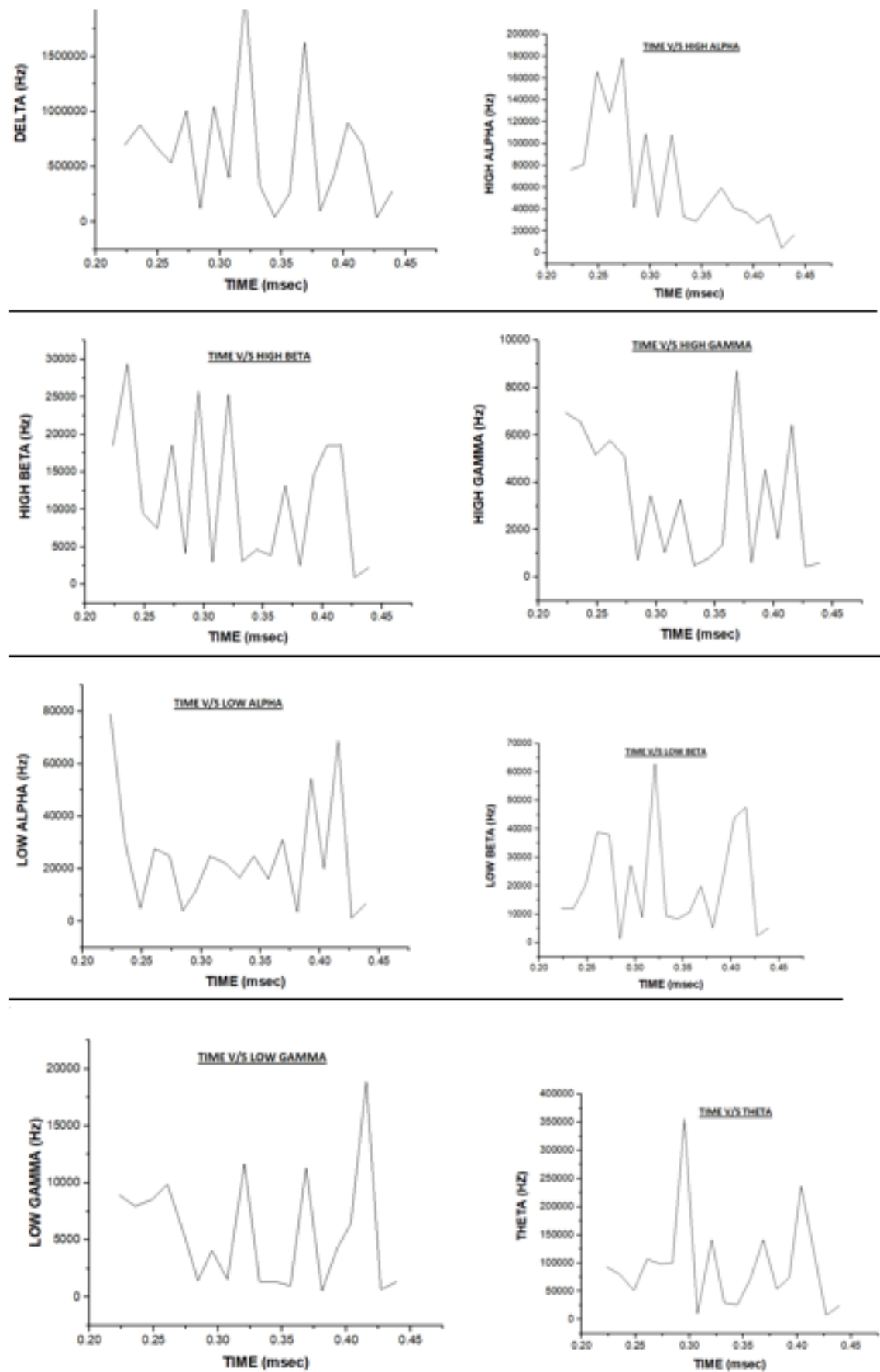

Fig. 2: Graphical output presentation of Meditation level data taken from human Brain

The mental state of the person has been observed from the values of low gamma, high gamma, high alpha, low alpha, high beta, low beta, delta and theta and the output graphs. The peak values of these graphs are greater than the standard values of each parameters. So, It can be predicted that the person's mental state is in meditated mode.

\section{CONCLUSION}

The collection of data from EEG can be used for research and development of various disease detection and the treatment based on the analyzed data can be started. By using EEG method, prediction of person's imagination can be done. For the better and accurate result the EEG is not only used in medical field but also used in crime detection sector and bio robotics and so on. The recognition of consciousness levels have been performed through the present concept. 


\section{ACKNOWLEDGMENT}

The authors of this paper are grateful for the blessings of almighty.

\section{REFERENCES}

[1] “A High Performance MEG Based BCI Using Single Trial Detection of Human Movement Intention”, Peter T. Lin, Kartikeya Sharma, Tom Holroyd, Harsha Battapady, Ding-Yu Fei and Ou Bai, Intech Openscience.

[2] "Brain computer interfacing: Applications and challenges", Sarah N. Abdulkader *, Ayman Atia, Mostafa-Sami M. Mostafa, Egyptian Informatics Journal (2015) $16,213-230$

[3] "Analysis of Electroencephalography (EEG) signals and Its Categorization- A Study", J Satheesh Kumar, P Bhubaneswari, Procedia Engineering 38 (2012) 2525 - 2536, Sciverse ScienceDirect, Elsevier.

[4] "Classification of EEG data for human mental state analysis using Random Forest Classifier", Damodar Reddy Edla, Kunal Mangalorekar, Gauri Dhavalikar, Shubham Dodia, Procedia Computer Science 132 (2018) 1523-1532, ScienceDirect, Elsevier.

[5] Your 5 Brainwaves: Delta, Theta, Alpha, Beta and Gamma, Lucid.

[6] E. Niedermeyer, F. H. Lopes da Silva. 1993. Electroencephalography: Basic principles, clinical applications and related fields, 3rd edition, Lippincott, Williams \& Wilkins, Philadelphia.

[7] H. L. Atwood, W. A. MacKay. 1989. Essentials of neurophysiology, B.C. Decker, Hamilton, Canada.

[8] F. S. Tyner, J. R. Knott. 1989. Fundamentals of EEG technology, Volume 1: Basic concepts and methods, Raven press, New York.

[9] P. L. Nunez. 1995. Neocortical Dynamics and Human EEG Rhythms, Oxford University Press, New York.

[10] The University of Sydney, Fundamentals of Biomedical Engineering, Electroencephalogram.

[11] “FUNDAMENTALS OF EEG MEASUREMENT”, M. Teplan, MEASUREMENT SCIENCE REVIEW, Volume 2, Section 2, 2002.

\section{AUTHORS}

First Author - Chaitali Bhattacharyya, B. Tech Student, Narula Institute of Technology.

Second Author - Trisha Paul, B. Tech Student, Narula Institute of Technology.

Third Author - Susmita Das, Assistant Professor, Narula Institute of Technology.

Fourth Author - Soutrik Karmakar, B. Tech Student, Narula Institute of Technology.

Fifth Author - Shyam Sundar Banerjee, B. Tech Student, Narula Institute of Technology.

Correspondence Author - Susmita Das, Assistant Professor, susmitad2011@ gmail.com, Narula Institute of Technology, 8017205403. 